\title{
Discovery of an Unusual High State in the Long-Term Light Curve of AM Herculis
}

\author{
S. KAFKA ${ }^{1}$ AND D. W. HOARD \\ Spitzer Science Center/Caltech, Pasadena, CA 91125 \\ Received 2009 September 6; accepted 2009 September 29; published 2009 November 12
}

\begin{abstract}
The magnetic cataclysmic variable archetype AM Herculis is known to display high and low photometric states, in which it switches from Roche lobe-overflow accretion (high) to wind accretion (low). The origin of this behavior is still unknown (although it is believed to be linked to the presence of starspots located near the L1 point on the secondary star), as are the specifics of duration and frequency of the two states. During the recent (2007) transition from an exceptionally long period of persistent low state behavior back to the high state, AM Her entered a short ( $\sim 2$ month) intermediate state, in which its brightness remained at $\sim 0.5$ mag fainter than the normal high-state level. Within the recorded history of AM Her, this was the first time the system displayed such behavior. Using data from the American Association of Variable Star Observers, we compare the characteristics of this faint high state to the subsequent normal high state, and a prior "failed high state," in which the system briefly reached a brightness level near that of the normal high state before rapidly returning to the low state. The distribution of magnitude values attained during each state, along with the shapes of the orbital light curve in each state, support a scenario in which the specific configuration of the accretion flow between the secondary star and the white dwarf must be rapidly established at the end of a low state, even before the mass transfer rate has reached the normal high-state level.
\end{abstract}

Online material: color figures

\section{INTRODUCTION}

Variable stars have been favorite targets of observers around the world for centuries. The periodic or irregular, and sometimes explosive, behavior reflected in their light curves intrigues and fascinates both professional and amateur astronomers alike. Systematic synoptic observations of variable stars started with the employment of robotic telescopes, dedicated for automated CCD monitoring (e.g., Honeycutt \& Turner 1992). At the same time, largely because of the dedication and commitment of amateur observers, we have a rich collection of long-term observations of the brightest objects, which reveal a diverse and uncanny group of behaviors among variable stars.

We focus here on the magnetic cataclysmic variable $(\mathrm{CV})$ AM Herculis, which is a popular observing target for both amateur and professional observers, with a pool of available data spanning more than a century. ${ }^{2}$ AM Her consists of a white dwarf (WD) and a Roche lobe-filling M4-5 main-sequence companion star (Kafka et al. 2005a) magnetically locked in a synchronous orbit with a period of $3.09 \mathrm{hr}$ (Kafka et al. 2005a

\footnotetext{
${ }^{1}$ Now at the Department of Terrestrial Magnetism, Carnegie Institution of Washington, Washington, DC 20015; skafka@dtm.ciw.edu.

${ }^{2}$ The first observation of AM Her in the AAVSO database is reported to have been taken in 1892 July.
}

and references therein). Tapia (1977) discovered that this system harbors a highly magnetic WD $(B \sim 13 \mathrm{MG})$, which made it the archetype of magnetic CVs, or polars.

The large collection of data about AM Her reveals a wealth of characteristics at all wavelengths. The most striking of these is that its long-term optical light curve displays high and low photometric states, in which the $V$ magnitude of the system alternates between $\sim 13.5$ and $\sim 15.4$. The high states are considered to be the "normal" accreting states of the system. The current established picture is that, during the high states the WD accretes from its low-mass companion via Roche lobe overflow through the inner Lagrangian point (L1). The accretion stream follows an initially ballistic trajectory, and eventually reaches one or more magnetic poles of the WD under the influence of the WD magnetic field lines. In the low state, the accretion rate drops to a very low level $\left(\sim 10^{-12} M_{\odot} \mathrm{yr}^{-1}\right.$; Gänsicke et al. 2006), consistent with accretion from the wind of the companion star instead of Roche lobe overflow through the L1 point. The long-term behavior of the high and low states of the system and the transitions to/from high states have been discussed extensively in the literature (e.g., see Kafka \& Honeycutt 2005, Kafka et al. 2005a).

High and low states are not unique characteristics of AM Her. They are present in all magnetic CVs with orbital periods less than $\sim 3 \mathrm{hr}$ and in many short-period nonmagnetic 
disk-accreting CVs, primarily in the orbital period range of 3$4 \mathrm{hr}$. At the same time, the cause of low states in these systems remains an open question. The current scenario supports that activity on the companion star may be responsible: when a large starspot on the M star, or spot group of sufficient size, crosses L1, the magnetic pressure of the spot(s) reduces the local gas pressure and accretion temporarily stops, leading to a drop in the luminosity of the system. Accretion resumes when the starspot/spot group dissipates or moves away from L1, and the brightness of the $\mathrm{CV}$ regains its original value (Livio \& Pringle 1994). The main advantages of this scenario are that it can explain the occurrence of low states in both disk and magnetic $\mathrm{CVs}$, and it is consistent with our understanding of the structure of the companion star in CVs at the orbital periods of systems for which the low states occur.

The calculated mean surface field for spots to occur on the companion star must be $\sim 10^{2} \mathrm{G}$ (Hameury et al. 1988). This theory is somewhat supported by observations (Hessman et al. 2000, Kafka \& Honeycutt 2005); however, it remains elusive in its details. For example, we are not aware of the processes that help reinstate the accretion stream at the end of a low state. Assuming that the transitions to/from high states are crossings of the penumbra and the umbra of a starspot at the L1 point (Honeycutt \& Kafka 2004), the long-term light curves of the system indicate that the usual accretion stream configuration is recovered almost instantaneously.

Recently, after a prolonged low state, during the transition back to the high state, AM Her lingered at $\sim 0.5$ mag fainter than its usual high-state brightness for approximately 2 months. This glitch in its usual state transition light curve behavior is strongly suggestive that AM Her was in an intermediate state, providing a short glimpse into the dynamics of the magneticallycontrolled accretion stream as it was reestablished in its normal high-state configuration.

\section{OBSERVATIONS}

Figure 1 presents 33 yr (1977-2009) of the recent long-term optical light curve of AM Her from the American Association of Variable Star Observers (AAVSO) and RoboScope ${ }^{3}$ databases. The light curve contains $5631 \mathrm{~V}$-band measurements from the AAVSO (starting in 1995), $2106 V$-band measurements from Roboscope (1991-2004), and an additional 14,836 "visual" measurements from the AAVSO. Typical uncertainties of the $V$-band data are $\approx 0.05 \mathrm{mag}$ for the AAVSO set and $\lesssim 0.02 \mathrm{mag}$ for the Roboscope set; the AAVSO visual data are precise to within $0.1 \mathrm{mag}$.

\footnotetext{
${ }^{3}$ RoboScope is a $0.41 \mathrm{~m}$ telescope in Indiana, equipped for automated, unattended differential CCD stellar photometry (Honeycutt \& Turner 1992). AM Her has been in the RoboScope database since 1991. RoboScope was in a maintenance mode during 2006; therefore, we complement its long-term light curve with data from the AAVSO database.
}

The system spends a large fraction of time at magnitude 13.5, accreting via L1 overflow onto its main magnetic pole. Although cyclotron emission from this pole is mostly visible in the near-IR, Kafka et al. (2005a) and Gänsicke et al. (2001) show that substantial cyclotron-generated flux is also present in the high-state $V$ light curve of AM Her. In the low state, AM Her remains at a quiescent $V$ magnitude of $~ 15.4$; however, its light curve is not featureless. Occasional short brightening events with amplitude of 0.2-0.6 mag and duration of several tens of minutes, or amplitude of $\sim 1$ mag and duration of up to several days (Kafka et al. 2005b), are present. The former are likely residual accretion events (de Martino et al. 1998) or flares on the secondary star (e.g., Shakhovskoy et al. 1993; Kalomeni \& Yakut 2008); the latter, while currently unexplained, are similar in appearance to the shorter flares, but with larger amplitude and longer duration (see Fig. 1, features C-F for examples of both of these transient events in the long-term AM Her light curve). Usually, low states last for about a year (or less). However, from 2003 September until 2007 June, AM Her was in an extended low state, interrupted only by a brief 2 month "failed" high-state episode in 2005 (Kafka \& Honeycutt 2005).

The particulars of the usual high states of AM Her are discussed in Gänsicke et al. (2001). When folded on the orbital period, the $V$ light curves are double-humped, with two equal peaks per orbit (near $\phi \sim 0.3$ and 0.7 ) corresponding to changing visibility of cyclotron emission from a single accreting pole. The shape and amplitude of the cyclotron emission feature are characteristic of the accreting region's shape, azimuth, and colatitude on the WD as well as the system inclination (Gänsicke et al. 2001). Over the long history of AM Her, accretion onto one or two poles, changing the shape of the orbital light curves, has also been reported (e.g., Gänsicke et al. 2001; Crosa et al. 1981). This is reflected in the phased $V$ light curves ${ }^{4}$ of $15 \mathrm{yr}$ of high-state data of the system (from RoboScope and AAVSO) presented in Figure 2. The failed high-state episode in 2005 (marked A in Fig. 1) lasted $\approx 45$ days in total, of which a total of $\approx 20$ days were spent in transition to/from the peak plateau level at $V \sim 13.7 \mathrm{mag}$. In comparison, the high states in AM Her usually last for more than 6 months and are $\sim 0.2$ mag brighter (see Fig. 1, and Kafka \& Honeycutt 2005). The short duration aside, the orbital light curve of the failed high state is similar in appearance to that of a normal high state, showing a roughly sinusoidal variation with two maxima per orbit (Fig. 2). Although the two peaks of the light curve are uneven in amplitude and shape, Figure 2 demonstrates that this is not a unique behavior for AM Her. Therefore, although the difference in the orbital light curve likely corresponds to a change of shape and/or location of the accreting region on the $\mathrm{WD}$, it does not explain the very short duration of this failed high state.

\footnotetext{
${ }^{4}$ For the phased light curves, we used the recent ephemeris of Kafka et al. 2005, $T_{0}=$ HJD 2, 446, 603.403(5) $+0.12892704(1) \times E$ which was derived using all the RoboScope data presented here.
} 

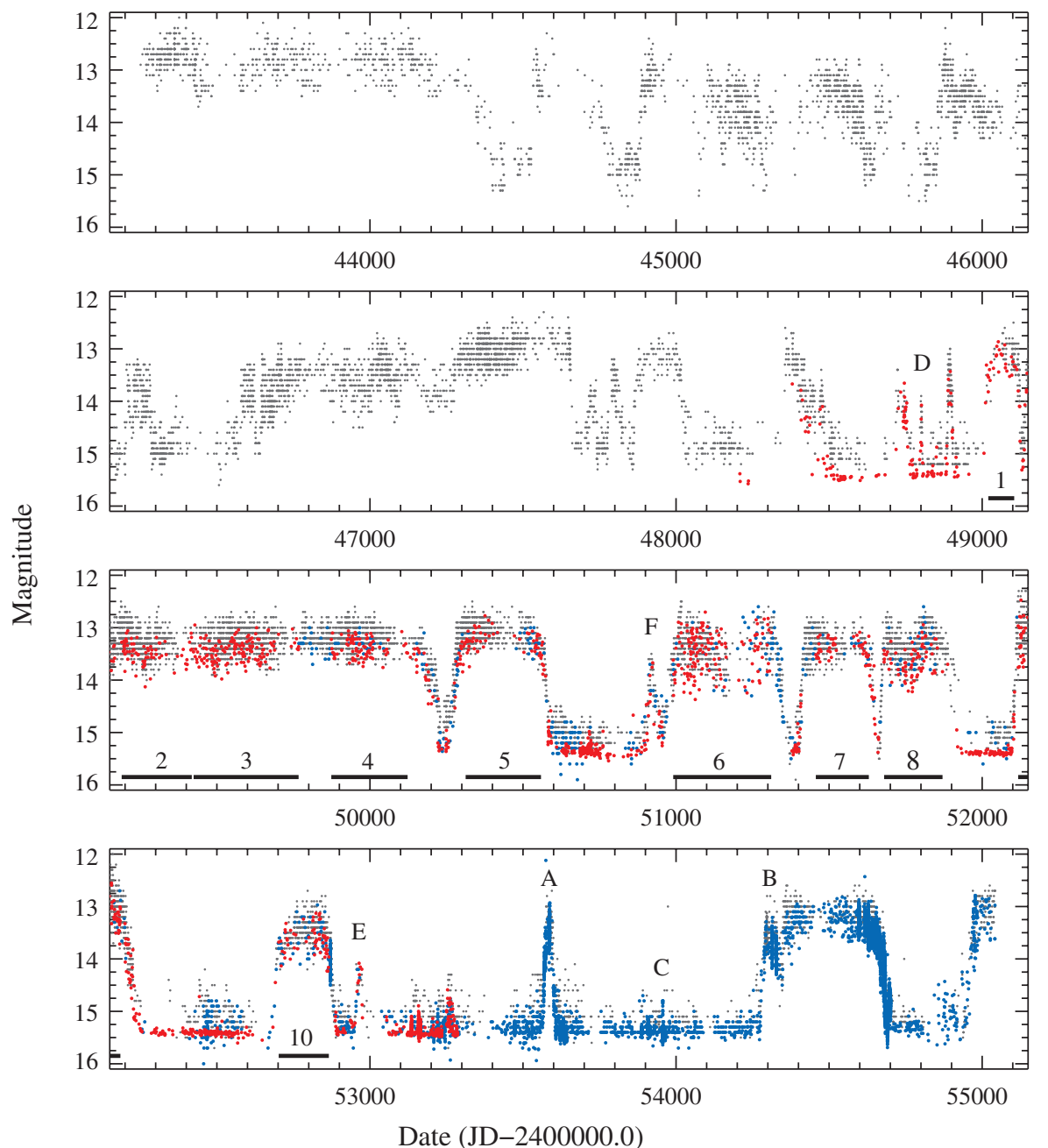

FIG. 1.-Long-term (1977-2009) light curve of AM Her showing visual (black) and $V$-band (blue) measurements from the AAVSO, and $V$-band (red) measurements from Roboscope. Several features are labeled: the failed high state (A), the faint high state (B), short flares (C, D), and long flares (E, F). The horizontal bars mark the high state regions presented as folded light curves in Fig. 2. (Note that high state 9 occurs at the border between the third panel from the top and the bottom panel and is not labeled.)

In more than 100 years of AM Her observations, the 20032007 low state is the longest in the history of the star. The system began to transition back to the high state in late 2007 June; however, it did not reach the usual high-state magnitude of $V \sim 13.7$. Up until early 2007 September, AM Her remained $\sim 0.5$ mag fainter than its regular high-state brightness, at an average magnitude of $\sim 14.0$. To add to the unusual behavior of this state transition, the overall brightness of the system dips slightly for $\sim 20$ days at the end of the faint high state, as though AM Her was about to head back into a low state (as in the failed high state), before recovering and continuing on to the normal high state (Fig. 3).

A quantitative demonstration of the uniqueness of the faint high state is shown in Figure 4, which is a histogram showing the distribution of all $V$-band magnitudes for AM Her from Figure 1 . The primary features are the two large peaks that cor- respond to the normal high and low photometric states. ${ }^{5}$ The faint high-state histogram has a similar width to the normal high state of the system; however, it is, on average, about half

\footnotetext{
${ }^{5}$ We do not see the weaker third peak near $V=13.8$ that was noted by Wu \& Kiss (2008) when they examined their histogram of the AAVSO visual data. This is likely a result of the fact that we have used only the more precise $V$-band data from the AAVSO (whereas the visual data used by Wu \& Kiss 2008 have a lower precision that essentially results in a quantization of values in increments of $0.1 \mathrm{mag}$, which could lead to spurious features in a histogram that is binned at the same step-size as the quantization of the data) and/or the fact that Wu \& Kiss (2008) also performed a rolling average of the data before binning into the histogram. Incidentally, we note that the low/high state duty cycle fractions quoted by Wu \& Kiss (2008) do not account for the fact that some of the variability in the long-term light curve is due to flaring activity on the secondary star, and not to bonafide state transitions. Their result in this regard only really delineates the difference between time spent brighter or fainter than $m_{v}=14.0$ mag regardless of the underlying reason for the $\mathrm{CV}$ to be at a particular brightness.
} 

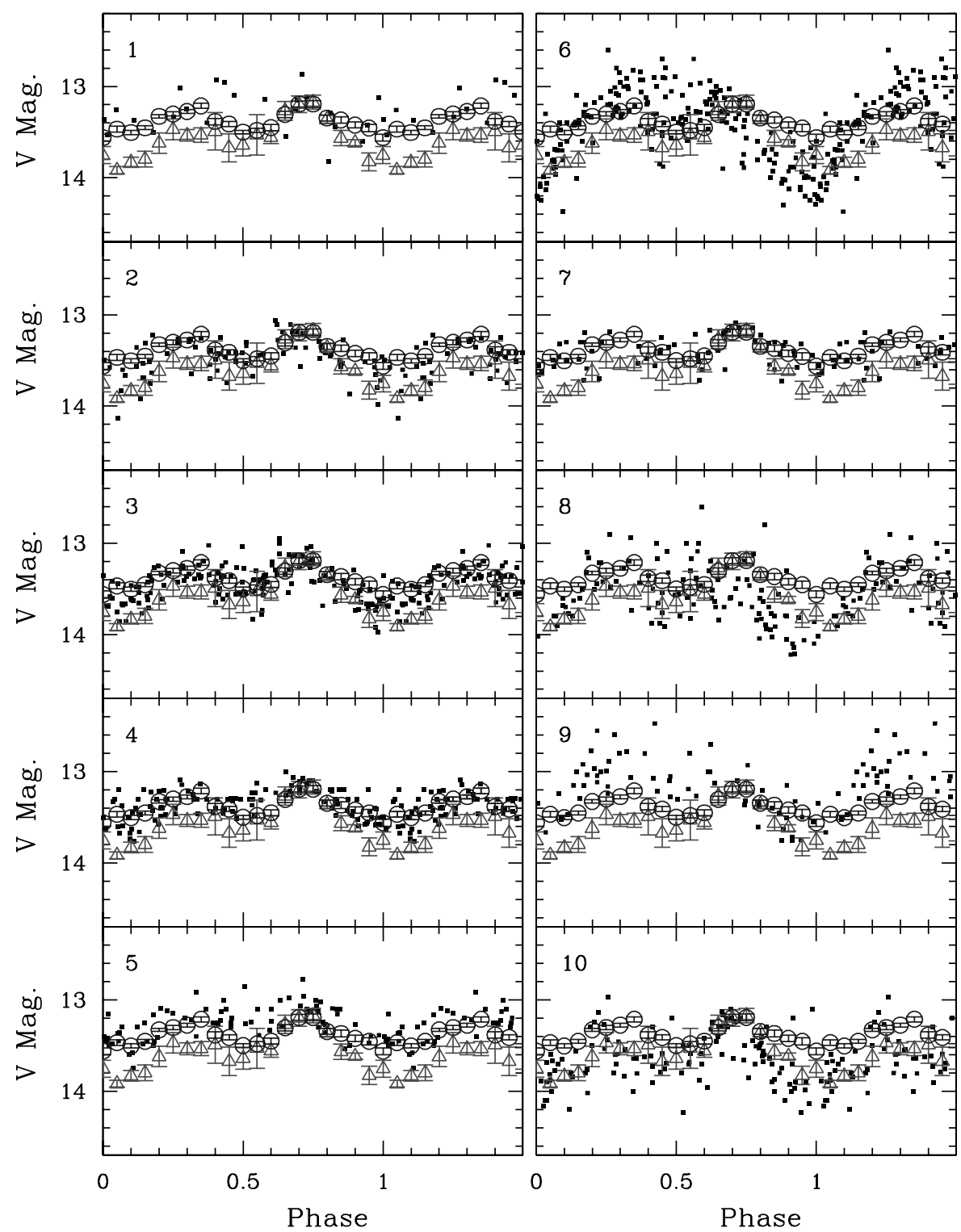

FIG. 2.-Phased high-state $V$ light curves of AM Her (filled black squares). Each panel corresponds to the numbered high-states (1-10) of Figure 1; open triangles are used for the average light curve of the bright part of the failed high state and open circles are used for the normal high state following the faint high state (also see Fig. 3). See the electronic edition of the PASP for a color version of this figure.

a magnitude fainter. The failed high-state magnitude distribution is broader, extending to fainter magnitudes comparable to the faint high state, and even slightly bimodal (discussed in $\S 3)$.

We note in passing that both the failed high state and the faint high state are also visible in the ROTSE IIId light curve of AM Her presented by Kalomeni \& Yakut (2008), although those authors did not explicitly comment on these features, as their analysis of the long-term light curve of AM Her was focused on short timescale variability, primarily during low states.

\section{DISCUSSION}

The transition from the end of the long low state to the faint high state in late 2007 is identical (in duration and speed) to any other transition between a low state and a normal high state (see Table 2 in Kafka \& Honeycutt 2005). This suggests that the mechanism that controls the occurrence of low-to-high state transitions is the same in the case of the 2007 transition as it was in previous ones. However, the temporary stop at the faint high state, before continuing on to the normal high state, suggests that the cause lies in the mechanism leading to the 
Time (days; faint high state)

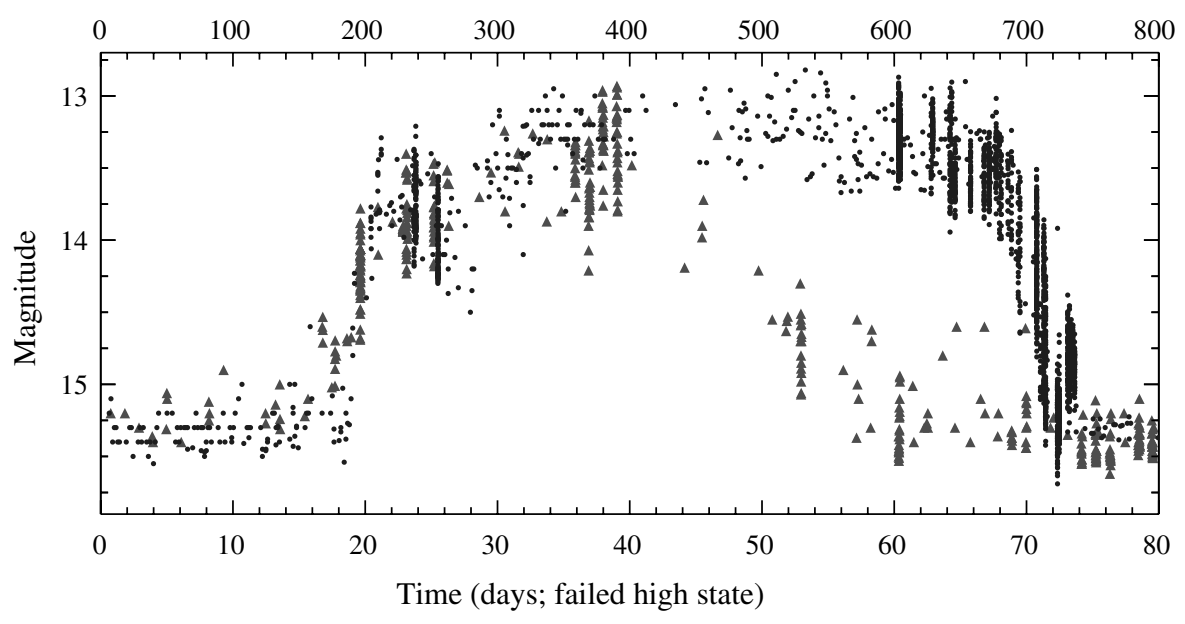

FIG. 3.-Faint high state and subsequent high state (circles) and failed high state (triangles) overlapped for comparison. The bottom axis corresponds to the failed high state and the top to the faint high state. The time axes have been scaled to demonstrate the similarities in brightness of time-dependent behavior (apart from duration) of the two states. See the electronic edition of the PASP for a color version of this figure.

reestablishment of the accretion stream following the resumption of mass overflow at L1.

Curiously, similar behavior is present only in the failed high state of 2005, whose development initially paused at a fainter plateau level (with magnitude comparable to that of the faint high state). Because of the sparseness of the data, it is not clear if the faint plateau in the failed high state was followed by a slight dip, as in the faint high state. The CV then proceeded to the normal high-state magnitude for a short time before it returned to the low state without establishing a high state of normal duration. In terms of overall behavior and brightness levels, this is similar to the onset of the faint high state through the beginning of the subsequent normal high state. The essential differences are that the failed high state episode was shorter in total duration (by a factor of $\approx 10$ ) and, of course, the failed high state did not lead to a subsequent high state. Figure 3 shows the $V$-band light curve data from the failed high state (region A in Fig. 1) and the faint high state/subsequent normal high state (region B in Fig. 1). The time axis of the faint high-state data has been compressed to match the light-curve evolution timescale of the failed high state to demonstrate the similarity in behavior described here.

The average phased light curve of the initial faint portion of the failed high state (JD 2,453,569.5-2,453,576.0) and of the faint high state (JD 2,454,281.0-2,454,330.0) are superimposed in Figure 5 with the average phased light curve of the normal high states that are shown in Figure 2. There is very good agreement between the light curves of the faint portion of the failed high state and the faint high state. On the other hand, while the bright portion of the failed high state (JD 2,453,576.0-2,453, 595.0) agrees with the usual high-state magnitude of AM Her (e.g., see Figs. 3 and 4), its average phased light curve has a different shape than that of the normal high state following the faint high state (see Fig. 2). The cyclotron emission peaks in the former are much more asymmetric than in the latter. Asymmetric cyclotron-dominated light curves in polars correspond to arc-shaped emission regions (Ferrario \& Wickramasinghe 1990).

The particulars of the accretion geometry (e.g., coupling region, accretion columns, accretion spot location on the WD, etc.) seem to be different in polars between the low and high

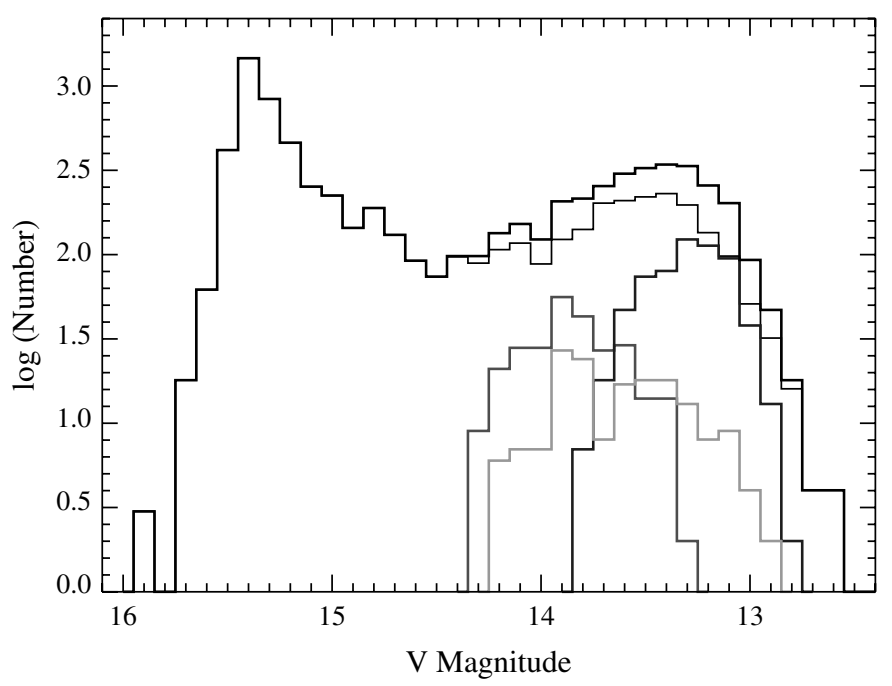

FIG. 4.- - Histogram of the $V$-band light curve data from Fig. 1 sorted into bins of width 0.1 mag. The thick black line shows the complete histogram, while the upper thin black line is the complete histogram from which the failed high state, faint high state, and subsequent normal high state have been subtracted. See the electronic edition of the PASP for a color version of this figure. 


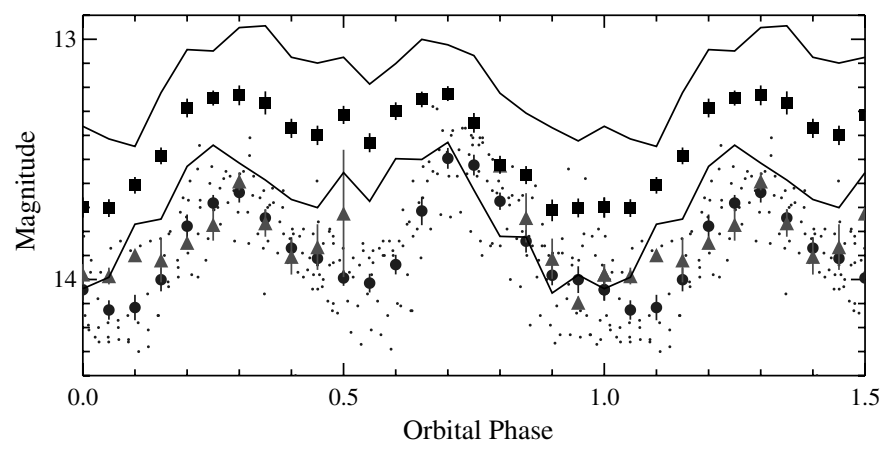

FIG. 5.-Phased light curves of AM Her; circles are the faint high state of the system, with large points showing the phase-binned light curve constructed from all the data (small points). The black squares are the average high-state RoboScope light curve. The solid lines show the range within which $75 \%$ of the RoboScope data lie. The triangles represent the average magnitude of the faint part of the failed high state (from AAVSO $V$ data). In this light curve, the bins centered at $\phi=0.55-0.75$ each contain $\leq 1$ data point, so are not plotted here. One anomalous data point at $V=12.12$ (visible above the label A in Fig. 1) has been removed from the bin average centered at $\phi=0.40$ because it is a $12 \sigma$ outlier compared to the other four data points in that bin. (It may have been misrecorded or affected by flaring activity). The bin with large error bars centered at $\phi=0.5$ contains only two data points but there is no justifiable reason to reject either of them. See the electronic edition of the PASP for a color version of this figure.

states, owing to the difference in both mass transfer rate and the geometry of the mass flow (wind vs. stream; Gänsicke et al. 2006). Assuming a radial infall of the stream toward the WD surface, Ferrario et al. (1989) derive that the coupling radius for polars ${ }^{6}$ is a function of the mass transfer rate $\dot{M}$ as: $r_{c} \propto \dot{M}^{-2 / 7}$. Between the high and low states of the system, a difference of $\sim 2.5$ magnitudes corresponds to a difference of more than 2 orders of magnitude in mass transfer rate (Hessman et al. 2000). The expectation is that the coupling region will be further away from the WD when the mass transfer rate is smaller. Using the correlation between $V$ magnitude and accretion flux provided in Figure 2 of Hessman et al. (2000), for average magnitudes of 13.4 in the normal high state and 13.9 in the faint part of the failed high state, the accretion flux rate is 1.5 times larger in the normal high state. In this case, we would expect a different coupling region of the stream in the faint high state, and a different accretion region structure onto the WD.

For AM Her, the spot colatitude changes by $\sim 15^{\circ}$ between the low and the high states (Gänsicke et al. 2006), reflecting changes in the coupling region of the star between the two states. When accretion stream mass transfer through the inner Lagrangian point is reinstated at the end of a low state, the polar generally rapidly reestablishes its high-state rate and configuration of accretion. As noted, this is likely to follow a different geometry than during the low state as a result of changes in

\footnotetext{
${ }^{6}$ Coupling radius is the distance from the WD surface at which the magnetic pressure first dominates over the ram pressure of the accretion stream
}

the location of the coupling region. In Figure 5 we present the phased light curve of the faint high state of AM Her. In this Figure, we also superimpose the average high-state RoboScope light curve and the average light curve from the faint part of the failed high state of 2005. The high-state light curve presents the typical sinusoidal modulation with two maxima per orbit (Kafka et al. 2005a) due to cyclotron emission from the main accreting pole (Gänsicke et al. 2001). Both the faint high state and normal high-state orbital light curves are similar in that they exhibit two dips and have an amplitude of $\sim 0.8$ mag. The striking similarity between those light curves suggests that the overall high-state accretion geometry is already established in the faint high state. Only the overall accretion rate is different, with the faint high state corresponding to a temporary hiatus in the climb of the mass transfer rate back to the normal high-state level.

Under the assumption that the Livio \& Pringle (1994) model of starspot-covering at L1 as the cause of low states is valid, Hessman et al. (2000) present a statistical model of the requisite coverage fraction and size distribution of the spots near L1 (see their Fig. 6). The patchy distribution of the spots and their large size compared to the accretion stream "nozzle" at L1 lead to the following possible scenario: the failed high state of 2005 resulted from a brief, yet full, recovery of the accretion stream, perhaps resulting from a temporary "gap" in the starspot distribution (e.g., a large spot that moved away from the L1 point). However, rather than completely dissipating (which would have lead to a normal high state), the starspot(s) returned or reformed, and the accretion flow was suppressed again. The post faintstate dip seen in the faint high state and possibly in the failed high state might signal the movement of the penumbra, followed by the umbra, of a large spot across the L1 point.

The faint high state occurred at the end of the longest recorded interval of persistent low-state behavior for AM Her, and apparently resulted from a glitch in the normally smooth transition from the low state. The long low state was punctuated only by a previous "failed attempt" to transition to the high state. Taken in the context of the starspot suppression model, this could imply that the long low state was an extended period of unusually high magnetic activity on the companion star in AM Her. While the causal significance of this behavior is not entirely clear (in part because the specific cause of low/high states in CVs is not yet fully understood), it highlights the fact that this particular low-to-high state transition in AM Her was different than previous transitions. However, the orbital phased light curves of the various states compared herein (i.e., failed high state, faint high state, normal high state) attest to one valuable conclusion that this unique photometric state has revealed: even before the mass transfer rate of the system has fully recovered to its high-state level, the accretion geometry (size and location of the accretion region on the WD and, presumably, the configuration of the accretion stream) has already been restored to the high-state configuration. Thus, resumption of Roche lobe overflow mass transfer at the end of a low state, even at an 
initially low rate of mass transfer during the transition to the stable high-state level, must very rapidly evolve into the stable high-state configuration.

We would like to thank Kent Honeycutt for making the RoboScope data available to us, and for constructive discussions. We acknowledge with thanks the variable star observations from the AAVSO International Database used in this research that were contributed by the following observers: Keith A. Graham, Stephen E. Robinson, Robert A. James, Francois M. Teyssier, Bill Goff, Ronald E. Zissell, Walter MacDonald, Tomas L. Gomez, Donn Starkey, John Blackwell, Zsolt Kereszty, Timothy R. Crawford, Ronald E. Royer, Don Wells, Kevin Kessler, Tom Michalik, Martin Nicholson, Danny
Scharnhorst, Roger Diethelm, Mauro Graziani, Andrzej Arminski, Richard P. Harvan, Gary Walker, Shawn Dvorak, Massimo Banfi, Thomas McDonagh, Dennis G. Hohman, Marino Fonovich, Michael Koppelman, John Grainger Observatory, Lewis Cook, Frank L. Scheder, Richard Sabo, Frans D.J. Nieuwenhout, Michael Simonsen, Jerry Doug West, Alain Bruno, Herve Chantegros, William Alexander, Aengus O'Fearghail, David A. Hurdis, Timothy Hager, William J. Aquino, Richard Huziak, Pere Closas, Marco Fiaschi, Alexandru Corlan, Barbara Harris, Ronald Baker, Richard Campbell, Kevin Krisciunas, Steve O'Connor, James Willams, William Dillon, David Scott Reynolds, Radu Corlan, Charles A. Suslavage, James A. Case, Horace Smith.

\section{REFERENCES}

Crosa, L., Szkody, P., Stokes, G., Swank, J., \& Wallerstein, G. 1981, ApJ, 247, 984

de Martino, D., et al. 1998, A\&A, 333, L31

Ferrario, L., \& Wickramasinghe, D. T. 1990, ApJ, 357, 582

Ferrario, L., Tuohy, I. R., \& Wickramasinghe, D. T. 1989, ApJ, 341,327

Gänsicke, B. T., Fischer, A., Silvotti, R., \& de Martino, D. 2001, A\&A, 372,557

Gänsicke, B. T., Long, K. S., Barstow, M. A., \& Hubeny, I. 2006, ApJ, 639, 1039

Hameury, J. M., King, A. R., Lasota, J. P., \& Ritter, H. 1988, MNRAS, 231, 535

Hessman, F. V., Gänsicke, B. T., \& Mattei, J. A. 2000, A\&A, 361, 952

Honeycutt, R. K., \& Turner, G. W. 1992, Robotic Telescopes in the 1990s, 34, 77
Honeycutt, R. K., \& Kafka, S. 2004, AJ, 128, 1279

Kafka, S., \& Honeycutt, R. K. 2005, AJ, 130, 742

Kafka, S., Honeycutt, R. K., \& Howell, S. B., \& Harrison, T. E. 2005a, AJ, 130, 2852

Kafka, S., Robertson, J., Honeycutt, R. K., \& Howell, S. B. 2005b, AJ, 129, 2411

Kalomeni, B., \& Yakut, K. 2008, AJ, 136, 2367

Wu, K., \& Kiss, L. L. 2008, A\&A, 481, 433

Livio, M., \& Pringle, J. E. 1994, ApJ, 427, 956

Shakhovskoy, N. M., Alexeev, I. Y., Andronov, I. L., \& Kolesnikov, S. V. 1993, Ann. Israel Phys. Soc. 10, Cataclysmic Variables and Related Physics, ed. O. Regev, \& G. Shaviv (Bristol: Institute of Physics) 237

Tapia, S. 1977, ApJ, 212, L125 